\title{
Antibiotic efficacy varies based on the infection model and treatment regimen for Pseudomonas aeruginosa
}

\author{
Cristina Cigana $\mathbb{1}^{1,2}$, Serena Ranucci ${ }^{1}$, Alice Rossi ${ }^{1}$, Ida De Fino ${ }^{1}$, \\ Medede Melessike ${ }^{1}$ and Alessandra Bragonzi, ${ }^{1,2}$
}

Affiliations: "Infections and Cystic Fibrosis Unit, Division of Immunology, Transplantation and Infectious Diseases, IRCCS San Raffaele Scientific Institute, Milano, Italy. ${ }^{2}$ Both authors contributed equally and share senior authorship.

Correspondence: Alessandra Bragonzi, Infections and Cystic Fibrosis Unit, Division of Immunology, Transplantation and Infectious Diseases, San Raffaele Scientific Institute, Milano, Italy.

E-mail: bragonzi.alessandraßhsr.it

@ERSpublications

Disease-specific animal models and treatment regimens are essential in order to optimise antiPseudomonas drug testing http://bit.ly/2ISfBiB

Cite this article as: Cigana C, Ranucci S, Rossi A, et al. Antibiotic efficacy varies based on the infection model and treatment regimen for Pseudomonas aeruginosa. Eur Respir J 2020; 55: 1802456 [https://doi.org/ 10.1183/13993003.02456-2018].

ABSTRACT Antibiotic discovery and preclinical testing are needed to combat the Pseudomonas aeruginosa health threat. Most frequently, antibiotic efficacy is tested in models of acute respiratory infection, with chronic pneumonia remaining largely unexplored. This approach generates serious concerns about the evaluation of treatment for chronically infected patients, and highlights the need for animal models that mimic the course of human disease.

In this study, the efficacy of the marketed antibacterial drugs tobramycin (TOB) and colistin (COL) was tested in murine models of acute and chronic P. aeruginosa pulmonary infection. Different administration routes (intranasal, aerosol or subcutaneous) and treatment schedules (soon or 7 days post-infection) were tested.

In the acute infection model, aerosol and subcutaneous administration of TOB reduced the bacterial burden and inflammatory response, while intranasal treatment showed modest efficacy. COL reduced the bacterial burden less effectively but dampened inflammation. Mice treated soon after chronic infection for 7 days with daily aerosol or subcutaneous administration of TOB showed higher and more rapid body weight recovery and reduced bacterial burden and inflammation than vehicle-treated mice. COL-treated mice showed no improvement in body weight or change in inflammation. Modest bacterial burden reduction was recorded only with aerosol COL administration. When treatment for chronic infection was commenced 7 days after infection, both $\mathrm{TOB}$ and $\mathrm{COL}$ failed to reduce $P$. aeruginosa burden and inflammation, or aid in recovery of body weight.

Our findings suggest that the animal model and treatment regimen should be carefully chosen based on the type of infection to assess antibiotic efficacy.

This article has supplementary material available from erj.ersjournals.com

Received: 28 Dec 2018 | Accepted after revision: 10 Oct 2019

Copyright $\odot$ ERS 2020. This version is distributed under the terms of the Creative Commons Attribution Non-Commercial Licence 4.0. 


\section{Introduction}

Worldwide, respiratory infection caused by Pseudomonas aeruginosa is one of the most critical healthcare burdens, with six million hospital admissions and over four million deaths annually $[1,2]$. In people with a weakened immune system, $P$. aeruginosa can cause acute respiratory infection leading to septicaemia and consequent fatality. In patients with cystic fibrosis (CF) and non-CF bronchiectasis, chronic life-threatening $P$. aeruginosa infections and inflammation lead to a progressive deterioration of pulmonary function [3, 4]. Despite the use of antibiotics, the control of $P$. aeruginosa infection remains perilously fragile owing to the rapid emergence of antibiotic resistance [5-7]. Thus, $P$. aeruginosa infections represent an especially relevant therapeutic challenge with an urgent need for the implementation of novel antimicrobial agents.

An essential prerequisite for drug development is the assessment of both safety and efficacy in animal models that mimic human disease. Although there are established guidelines that specify appropriate animal models and parameters relevant for assessing antibiotic safety, there is a lack of well-accepted guidelines for efficacy testing $[8,9]$. The most commonly used animal model for evaluating the efficacy of antimicrobial agents against $P$. aeruginosa reproduces the acute respiratory infection in immunocompetent or neutropenic mice $[10,11]$. However, these models are characterised by a rapid disease course and, while useful as acute respiratory infection models, they lack any characteristics of the events triggered in an established chronic infection. Furthermore, the use of neutropenic mice does not allow the host response to be assessed as a potential determinant of drug efficacy.

Although mouse models of chronic $P$. aeruginosa pneumonia have been generated to mimic the advanced stage of lung pathology in humans [10, 12-15], these models are technically more complicated than acute infections and pose several challenges. Chronic infection in these models is usually established by including bacterial cells in immobilising agents where they appear to grow in microcolonies under micro-aerobic/anaerobic conditions, as they do in the mucus of CF patients [16]. These models present major challenges related to the low $P$. aeruginosa burden and percentage of infected mice weeks after infection [12]. Although they are useful in evaluating the impact of repeated administrations in the initiation and progression of the chronic infection, these models have elevated preclinical costs. Because they are not used systematically and specifically on a preclinical platform that supports antibacterial efficacy testing, their predictive value has not been fully established. More importantly, it is unclear whether the use of a model of chronic infection in antibiotic efficacy testing against $P$. aeruginosa is advantageous and necessary compared to the acute infection model. In addition, the impact that the route of administration and the treatment schedule might have in drug efficacy is largely unknown.

In this study, we aim to define the differences between mouse models and treatment regimens in the efficacy of two marketed antibacterial drugs of the aminoglycosides and polymyxin classes, tobramycin (TOB) and colistin (COL) respectively. We designed the study to 1) evaluate treatment efficacy in murine models of acute and chronic $P$. aeruginosa respiratory infection; 2) determine if administration routes have an impact on pathogen clearance and host response using aerosol (aero), intranasal (i.n.) and subcutaneous (s.c.) delivery; and 3) determine if different treatment schedules affect efficacy by comparing treatment administered soon after infection to treatment administered during chronic colonisation.

\section{Methods}

\section{Ethics statement}

Animal studies adhered to the Italian Ministry of Health guidelines for the use and care of experimental animals (IACUC \#733 and \#878). Research with the P. aeruginosa multidrug-resistant (MDR)-RP73 isolate from a $\mathrm{CF}$ individual and storage of biological materials were approved by the Ethics Commission of Hannover Medical School, Germany.

\section{Bacterial strains}

P. aeruginosa strains included PAO1 and MDR-RP73 strains isolated from a CF patient's airways. Minimum inhibitory concentrations (MICs) of TOB and COL were previously determined (supplementary table S1) [17].

\section{Mouse model}

Immunocompetent C57BL/6NCrlBR male mice (8-10 weeks; Charles River Laboratories, Calco, Italy) were challenged with $1 \times 10^{6}$ colony-forming units (CFUs) of the planktonic PAO1 strain for acute infection or $5 \times 10^{5}$ CFUs of the MDR-RP73 strain embedded in agar beads for chronic infection by intratracheal administration [12-14]. Mice were treated with TOB, COL or vehicle (water) by local administration using a Penn-Century MicroSprayer ${ }^{\circledast}$ Aerosoliser (aero) or by i.n. or systemic s.c. administration (figure 1). Body weight was monitored daily. Lung CFUs and cell counts in the bronchoalveolar lavage fluid (BALF) were 


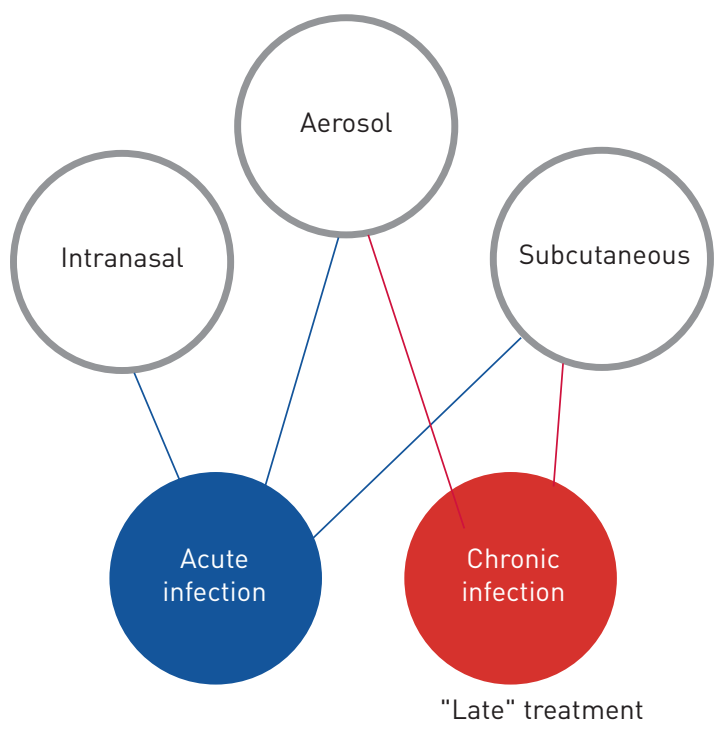

"Early" treatment

\section{Chronic}

\section{Chronic}

Acute

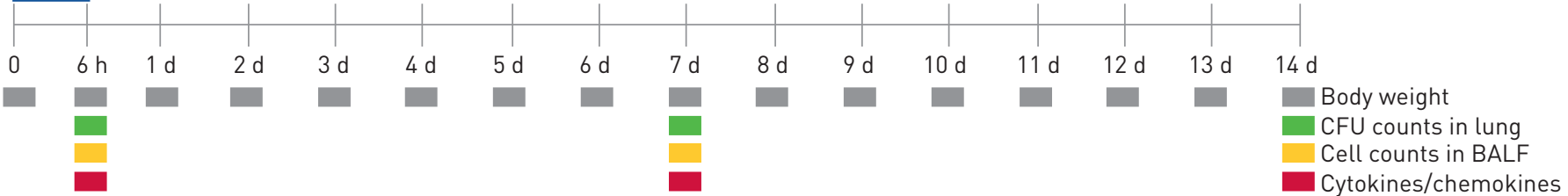

FIGURE 1 A schematic representation of the antibiotic treatment schedule and analysis in murine models of acute and chronic Pseudomonas aeruginosa infection. At day 0 , mice were infected with $P$. aeruginosa planktonic cells to mimic acute infection or with $P$. aeruginosa embedded in agar beads to achieve long-term chronic infection. In the acute infection model, the treatment schedule used with the antibiotics was a single dose administered 5 min after infection by aerosol, intranasal or subcutaneous routes. In the chronic infection model, the treatment started 5 min ("early" treatment) or 7 days ("late" treatment) after infection, with repeated daily doses for 7 days. Read-outs of the disease progression were body weight changes, colony-forming units (CFUs), total and differential cells, and cytokines/chemokines assayed at the time of death (at $6 \mathrm{~h}$ for acute infection or after 7 days of treatment for chronic infection). d: days; BALF: bronchoalveolar lavage fluid.

analysed as described previously $[13,18,19]$. Cytokine/chemokine levels were measured in the supernatant of lung homogenates by Bioplex Assay (Bio-Rad Laboratories, Segrate, Italy). Pharmacokinetic (PK) profiles of TOB and COL in the lungs and plasma of $P$. aeruginosa-infected mice were evaluated by high-performance liquid chromatography-tandem mass spectrometry. Additional details in accordance with the Animal Research: Reporting of In Vivo Experiments guidelines [20] are reported in the supplementary material.

\section{Statistics}

Statistical analyses were performed with GraphPad Prism (GraphPad Software, Inc., San Diego, CA, USA) using a two-way ANOVA with Bonferroni's multiple comparison test for body weight changes and one-way ANOVA with Bonferroni's multiple comparison test for the other readouts. Outlier data, identified by Grubbs' test, were excluded from the analysis.

\section{Results}

Treatment efficacy is affected by the route of administration and type of antibiotic in acute $\mathrm{P}$. aeruginosa lung infection

C57BL/6NCrlBR mice were challenged with the planktonic $P$. aeruginosa PAO1 strain by intratracheal inoculation to induce acute infection. Local treatment via the aero or i.n. route with $2 \mathrm{mg} \cdot \mathrm{kg}^{-1} \mathrm{TOB}$ or $1 \mathrm{mg} \cdot \mathrm{kg}^{-1} \mathrm{COL}$ and systemic treatment via the s.c. route with $20 \mathrm{mg} \cdot \mathrm{kg}^{-1} \mathrm{TOB}$ or $10 \mathrm{mg} \cdot \mathrm{kg}^{-1} \mathrm{COL}$ started soon $(5 \mathrm{~min}$ ) after infection (figure 1). TOB and COL were dosed to keep the same dose to MIC ratio (supplementary table S1) and local to systemic ratio, based on previous studies [17]. A single dose of TOB was significantly effective at $6 \mathrm{~h}$ post-treatment, with a $4-\log _{10}$ reduction in lung CFUs on aero 
administration and a $3-\log _{10}$ reduction on s.c. administration when compared to CFUs observed with vehicle (figure $2 \mathrm{a}$ ). It is worth noting that TOB administered via either the aero or s.c. route was able to completely clear the infection in at least half of the treated mice (supplementary table S2). TOB displayed limited ability for bacterial clearance and low efficacy following i.n. administration, with a $1-\log _{10} \mathrm{CFU}$ reduction compared to CFUs observed with vehicle. COL was generally less effective than TOB in reducing the bacterial burden (figure $2 \mathrm{~b}$ ). COL-treated mice showed a significantly reduced bacterial

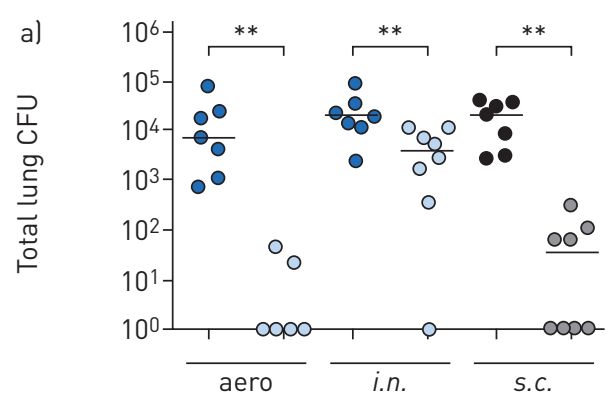

- Vehicle

O TOB $2 \mathrm{mg} \cdot \mathrm{kg}^{-1}$

- Vehicle

- TOB $20 \mathrm{mg} \cdot \mathrm{kg}^{-1}$
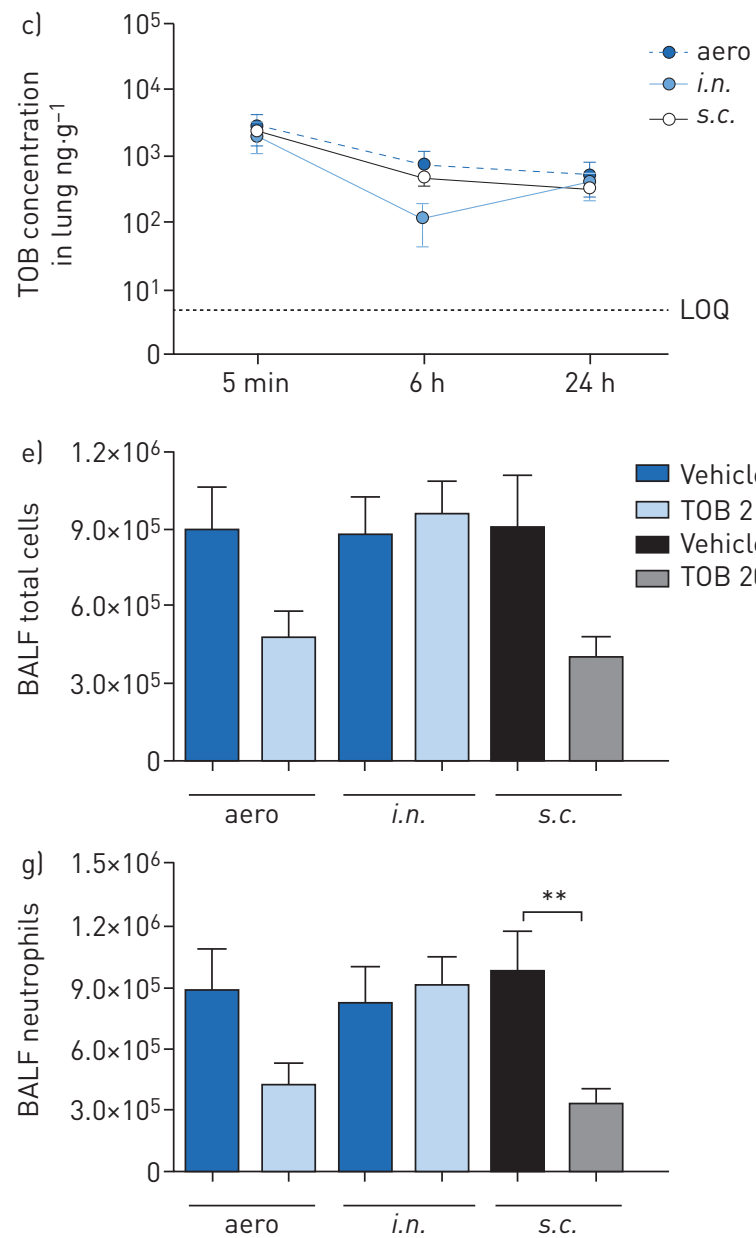
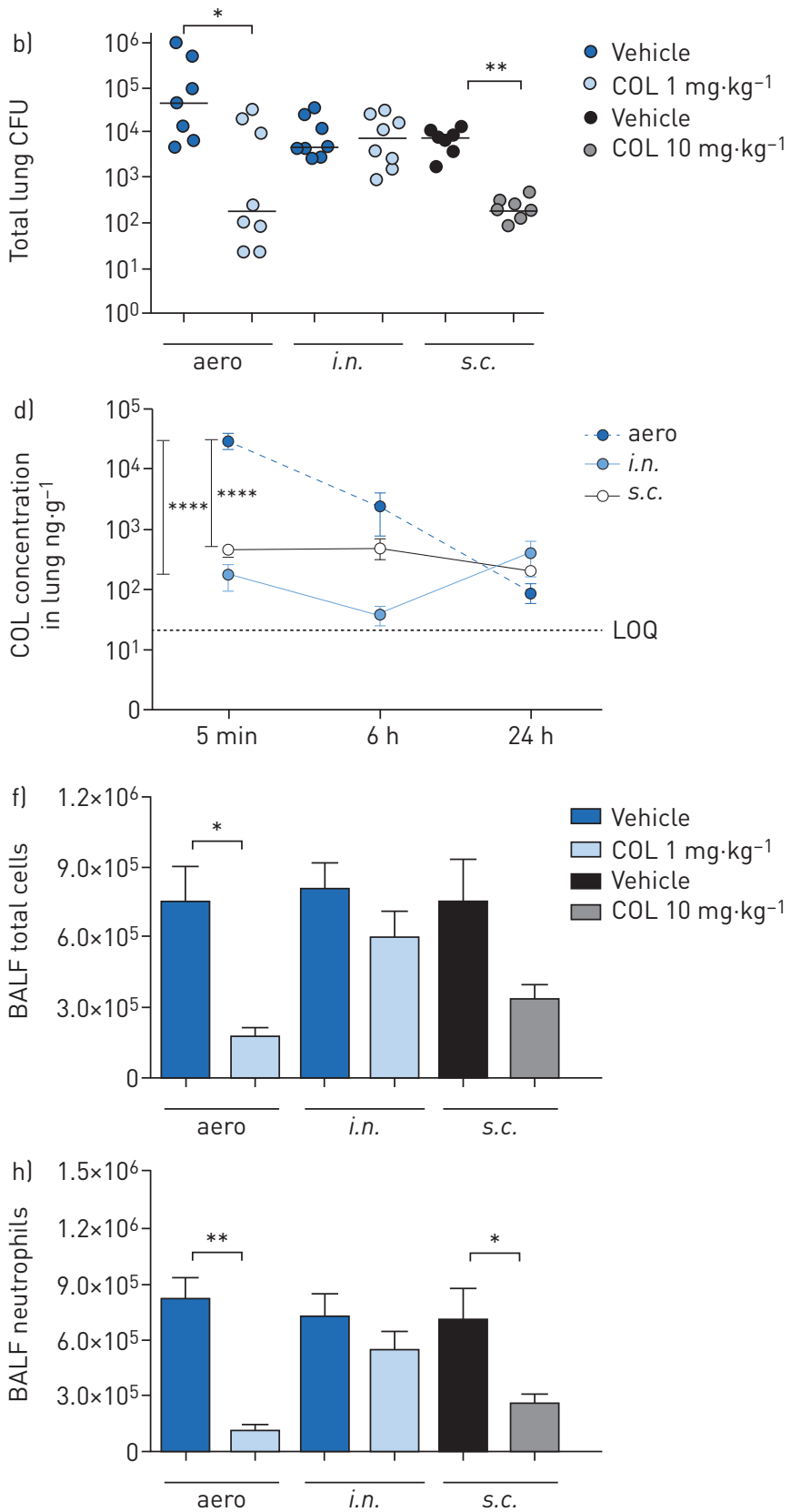

FIGURE 2 Efficacy and pharmacokinetics of tobramycin (TOB) (a, c, e, g) and colistin (COL) (b, d, f, h) in a mouse model of acute Pseudomonas aeruginosa airway infection. C57BL/6 male mice laged 8-10 weeks) received intratracheal inoculations with $1 \times 10^{6}$ colony-forming units (CFUs) of planktonic PA01. 5 min after infection, $2 \mathrm{mg} \cdot \mathrm{kg}^{-1} \mathrm{TOB}, 1 \mathrm{mg} \cdot \mathrm{kg}^{-1} \mathrm{COL}$ or vehicle was administered via an aerosoliser (aero) or the intranasal (i.n.) route. Alternatively, $20 \mathrm{mg} \cdot \mathrm{kg}^{-1} \mathrm{TOB}, 10 \mathrm{mg} \cdot \mathrm{kg}^{-1} \mathrm{COL}$ or vehicle was administered via the subcutaneous (s.c.) route. a-h) After $6 \mathrm{~h}$, the mice were killed, bronchoalveolar lavage fluid (BALF) was collected, and the lungs were excised, homogenised and plated on tryptic soy agar to determine the bacterial burden $(\mathrm{a}, \mathrm{b})$. Each dot represents CFUs per lung from one mouse, and horizontal lines represent the median values. $\mathrm{c}$, d) For pharmacokinetics analysis, mice were killed $5 \mathrm{~min}, 6 \mathrm{~h}$ and $24 \mathrm{~h}$ after treatment, and the concentration of antibiotics was measured in the lung and plasma (supplementary figure S1). The limits of quantification (LOQs) are indicated. e, f) Total cell and g, h) neutrophil counts were performed in BALF. Data are presented as mean \pm SEM pooled from two independent experiments ( $n=7-8$ for efficacy, and $n=4-8$ for pharmacokinetics). *: $p<0.05 ;{ }^{* *}: p<0.01{ }^{* * * *}: p<0.0001$. 
burden in the lungs, with a $2-\log _{10}$ CFU reduction after aero and s.c. administration compared to CFUs observed with vehicle, but complete clearance was not observed. No reduction in lung CFUs was observed with COL following i.n. administration. In summary, in acute infection, both TOB and COL treatments administered via the aero and s.c. routes reduced bacterial burden, with COL being less effective than TOB. Administration via the i.n. route was the least effective of all three routes, with no demonstrable efficacy for COL.

To interpret these results further, we examined the PK profiles of TOB and COL in the lungs and plasma of $P$. aeruginosa-infected mice. A large quantity of TOB was concentrated and retained in the lung following both local (aero or i.n.) and systemic (s.c.) administration for up to $24 \mathrm{~h}$ after administration (figure 2c). These data support the study design and the local to systemic ratios. In contrast, COL concentration in the lung was significantly higher following aero administration than with s.c. or i.n. administration (figure $2 \mathrm{~d}$ ). When viewed in relation to the decreased efficacy of TOB and COL (figure $2 a, b$ ), these findings do not support a direct correlation between efficacy (CFUs per lung) and drug concentration in the lung (PK). As expected, plasma concentrations of both TOB and COL were significantly higher with systemic (s.c.) than with local (aero or i.n.) administration (supplementary figure S1a and b).

To define the effect of TOB and COL on the airway inflammatory response, we measured leukocyte recruitment in the BALF and cytokine/chemokine concentrations in lung homogenates. $6 \mathrm{~h}$ after acute PAO1 infection, mice treated with TOB by the aero or s.c. routes had fewer total cell counts (figure 2e) than vehicle-treated mice, indicating a reduction in inflammation. Although COL was generally less effective than TOB in terms of bacterial burden reduction, it influenced the recruitment of leukocytes in the BALF following aero or s.c. administration (figure 2f). Neutrophil counts decreased after both TOB (figure $2 \mathrm{~g}$ ) and COL (figure $2 \mathrm{~h}$ ) treatments via the aero and s.c. routes but were unaffected by treatment via the i.n. route. Macrophage counts remained unchanged following aero or i.n. administration but increased after s.c. treatment with both TOB and COL (supplementary figure S2a, b).

Cytokine/chemokine profiles showed that granulocyte colony-stimulating factor (G-CSF) and keratinocyte chemoattractant $(\mathrm{KC})$ were consistently and significantly reduced in lungs of mice treated with both TOB and COL by aero and s.c. administration (table 1). Additionally, macrophage inflammatory protein-1 $\beta$ (MIP-1 $\beta)$ and tumour necrosis factor- $\alpha$ (TNF- $\alpha$ ) were significantly reduced by both TOB and COL treatments, although to a lesser extent. Reduction of these cytokines/chemokines to a similar extent with both TOB and COL, despite COL displaying lower efficacy (CFUs per lung) than TOB, suggests the lack of a direct correlation between the cytokine/chemokine response and bacterial burden. Other cytokines/ chemokines that were affected by the type of antibiotic and route of administration included interleukin (IL)-1 $\alpha$, IL-6, IL-12p70, granulocyte-macrophage colony-stimulating factor (GM-CSF), monocyte chemoattractant protein-1 (MCP-1), MIP-1 $\alpha$ and RANTES.

\section{Efficacy is affected by the treatment schedule and type of antibiotic in chronic P. aeruginosa lung infection}

To mimic a chronic infection similar to what is typically established in the lungs of CF patients, mice received intratracheal inoculations with the P. aeruginosa MDR-RP73 strain embedded in agar beads [13]. Administration via the aero route with $16 \mathrm{mg} \cdot \mathrm{kg}^{-1} \mathrm{TOB}$ or $1 \mathrm{mg} \cdot \mathrm{kg}^{-1} \mathrm{COL}$ (supplementary table S1) was started 5 min ("early" treatment; figure 3) or 7 days ("late" treatment; figure 4) after infection and was repeated daily for seven administrations (figure 1). The doses used for s.c. administration were $160 \mathrm{mg} \cdot \mathrm{kg}^{-1}$ for TOB and $10 \mathrm{mg} \cdot \mathrm{kg}^{-1}$ for COL, based on dose to MIC ratio.

Over the course of 7 days of "early" treatment, TOB-treated mice exhibited significantly less loss and faster recovery of body weight than vehicle-treated mice (figure 3a). Compared to vehicle-treated mice, TOB-treated mice showed a significant reduction in bacterial burden, with most of the mice clearing the infection following either aero or s.c. administration (figure 3c, supplementary table S2). TOB treatment significantly reduced total cell counts in the BALF, including neutrophils, when compared to treatment with vehicle, indicating a reduction in inflammation (figure $3 \mathrm{e}, \mathrm{g}$ ). Macrophage counts were reduced in mice treated with TOB by s.c. administration (supplementary figure S2a). IL-1 $\alpha$, IL-6, IL-12p40, IL-17A, MIP-1 $\alpha$, RANTES and TNF- $\alpha$ were significantly reduced by both the aero and s.c. administration routes (table 2). IL-2 and KC were significantly decreased, while IL-9 and IL-13 increased only with aero TOB administration. MIP-1 $\beta$ was significantly reduced, while IL-5 increased exclusively with s.c. TOB administration. Thus, when administered soon after infection, TOB was effective in reducing bacterial burden and inflammation in the lungs by both aero and s.c. routes in the chronic infection model.

Over the course of 7 days of "early" treatment, COL-treated mice lost and recovered body weight comparably to vehicle-treated mice (figure $3 b$ ). Compared to treatment with the vehicle, treatment with 
TABLE 1 Cytokine/chemokine concentrations following acute Pseudomonas aeruginosa airway infection and antibiotic treatment

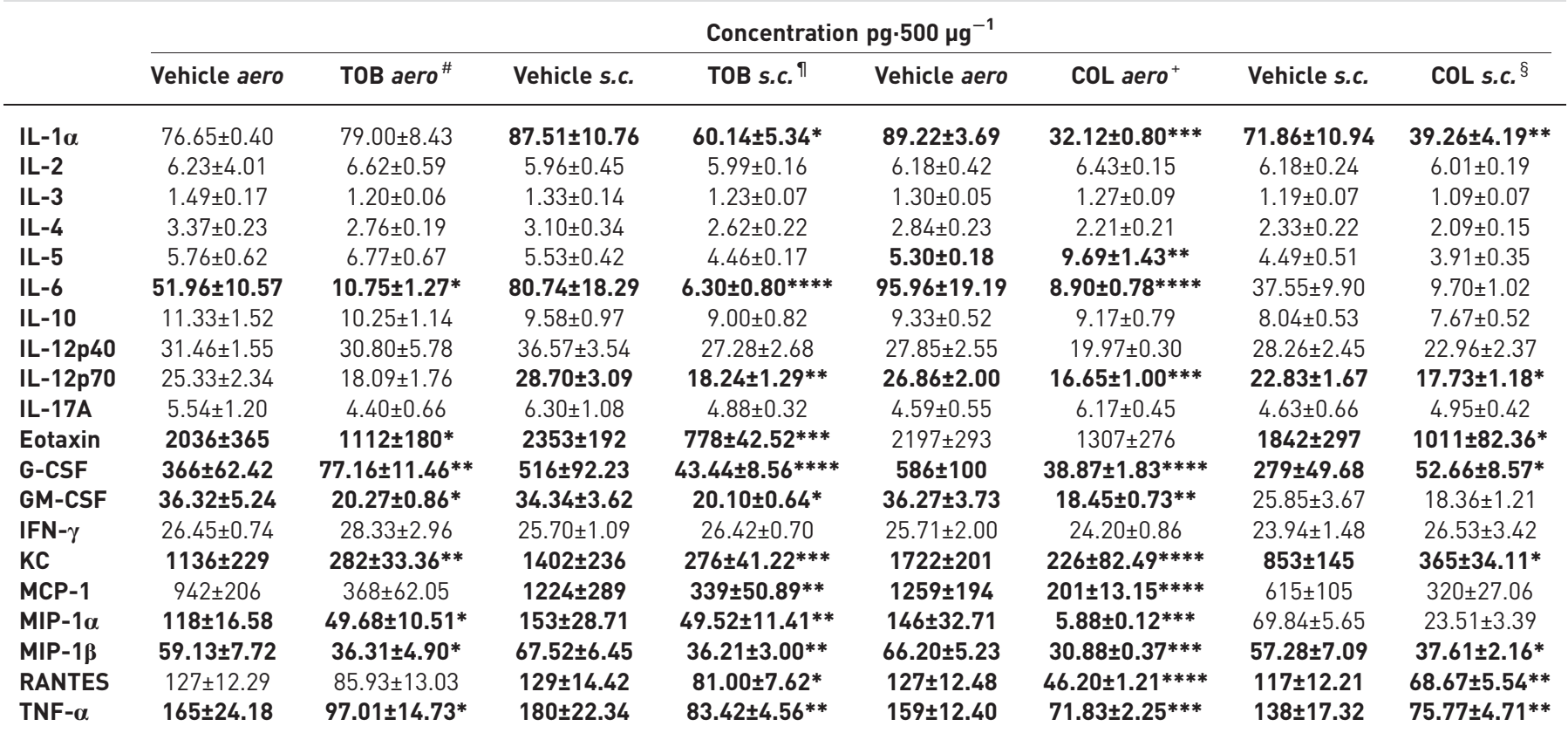

Data are presented as mean \pm SEM pooled from two independent experiments $(n=4-5)$. Statistical significance determined by one-way ANOVA with Bonferroni's multiple comparison test, with significant differences between treatment and vehicle highlighted in bold. aero: delivery using an aerosoliser; TOB: tobramycin; s.c.: subcutaneous; COL: colistin; IL: interleukin; G-CSF: granulocyte colony-stimulating factor; GM-CSF: granulocyte-macrophage colony-stimulating factor; IFN: interferon; KC: keratinocyte chemoattractant; MCP: monocyte chemoattractant protein; MIP: macrophage inflammatory protein; TNF: tumour necrosis factor; ${ }^{\#}: 2 \mathrm{mg} \cdot \mathrm{kg}^{-1} ;{ }^{\uparrow}: 20 \mathrm{mg} \cdot \mathrm{kg}^{-1} ;{ }^{+}: 1 \mathrm{mg} \cdot \mathrm{kg}^{-1} ; \S^{\S}: 10 \mathrm{mg} \cdot \mathrm{kg}^{-1}$; ${ }^{*}: p<0.05 ;^{* *}: p<0.01 ;^{* * *}: p<0.001 ;{ }^{* * *}: p<0.0001$.

COL by aero administration significantly reduced the bacterial burden (figure $3 \mathrm{~d}$ ), while s.c. administration was not effective. Total and differential cell counts in the BALF showed a trend for reduction, which was not statistically significant for either administration route (figure $3 \mathrm{f}, \mathrm{h}$ and supplementary figure S2b). Accordingly, only a few cytokines/chemokines, including IL-12p40, IL-17A, eotaxin, MIP-1 $\alpha$ and RANTES, were significantly decreased following COL treatment via the aero route, while no inflammatory mediators were reduced following s.c. administration (table 2). Thus, when administered soon after infection, COL was effective in reducing bacterial burden and a few cytokines/chemokines in the lungs via the aero route, with no major effects observed via the s.c. route of administration in the chronic infection model.

When treatments were started 7 days after infection ("late" treatment), body weight recovery did not differ between TOB- and vehicle-treated mice (figure 4a). TOB administered via the aero or s.c. routes failed to reduce the bacterial burden (figure 4c) or cytokine/chemokine levels (table 3). TOB administered via the aero route resulted in significantly higher total cell counts (figure $4 \mathrm{e}, \mathrm{g}$ ), predominantly of macrophages (supplementary figure S3a), when compared to vehicle-treated mice. TOB administered via the s.c. route did not affect inflammation.

Similar to the results obtained with "late" TOB treatment, the loss or recovery of body weight (figure $4 \mathrm{~b}$ ), the bacterial burden (figure $4 \mathrm{~d}$ ) and inflammatory cell recruitment (figure $4 \mathrm{f}, \mathrm{h}$ and supplementary figure $\mathrm{S} 3 \mathrm{~b}$ ) did not differ between "late" COL- and vehicle-treated mice. Cytokine/chemokine profiles were unchanged between "late" COL- and vehicle-treated mice, supporting a lack of efficacy (table 3). Our results indicate that treatment by TOB and COL in the chronic infection model was only effective when the antibiotics were administered soon after infection.

\section{Discussion}

Antibacterial efficacy is affected by the route of administration, type of antibiotic and infection model

First, we used the acute infection model established by direct intratracheal administration of the planktonic reference strain PAO1 to evaluate the efficacy of the antibacterial agents TOB and COL delivered by local 

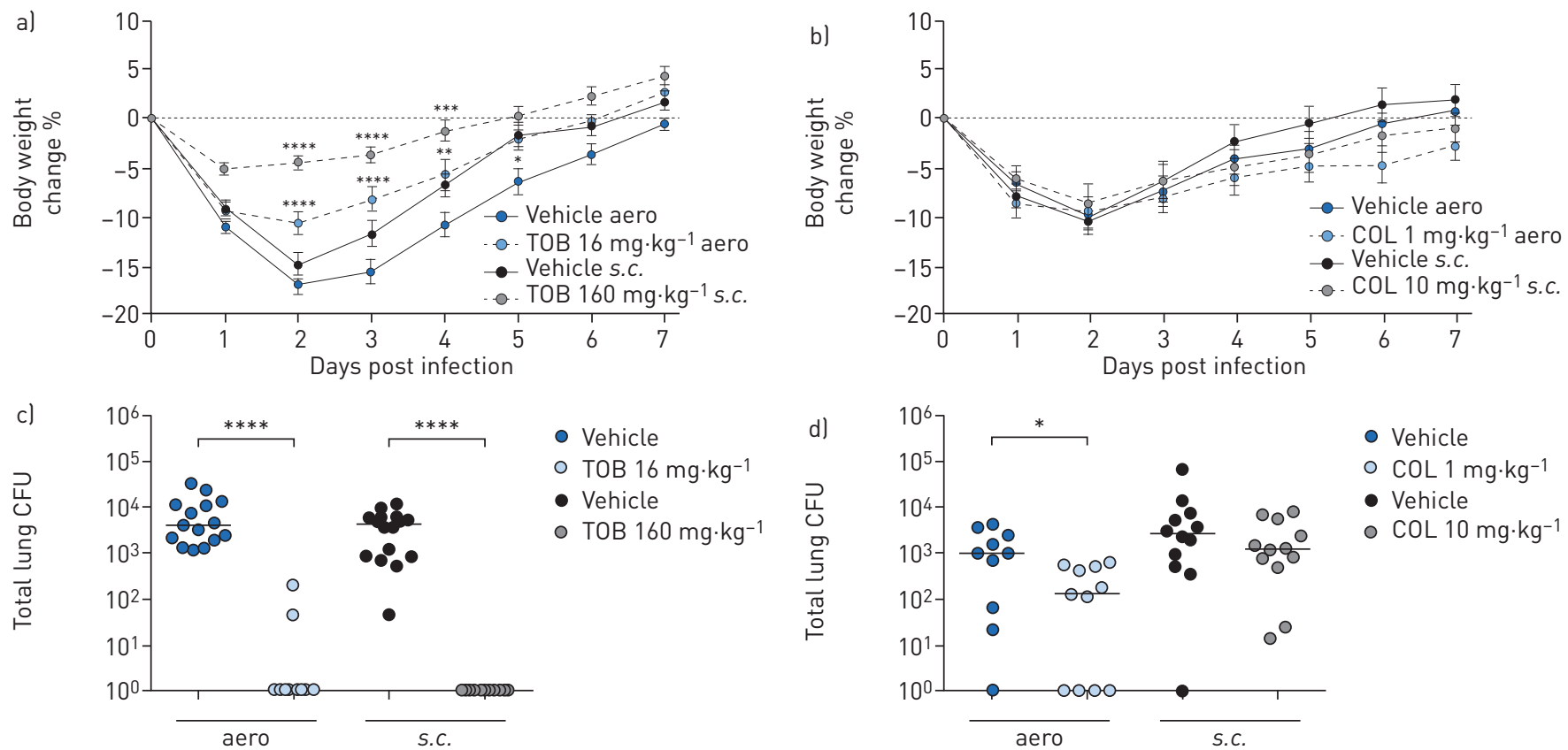

- Vehicle

- TOB $16 \mathrm{mg} \cdot \mathrm{kg}^{-1}$

- Vehicle

- TOB $160 \mathrm{mg} \cdot \mathrm{kg}^{-1}$

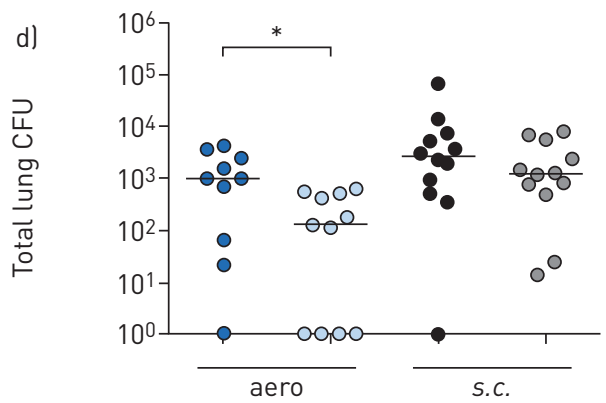

- Vehicle

- COL $1 \mathrm{mg} \cdot \mathrm{kg}^{-1}$

- Vehicle

- COL $10 \mathrm{mg} \cdot \mathrm{kg}^{-1}$
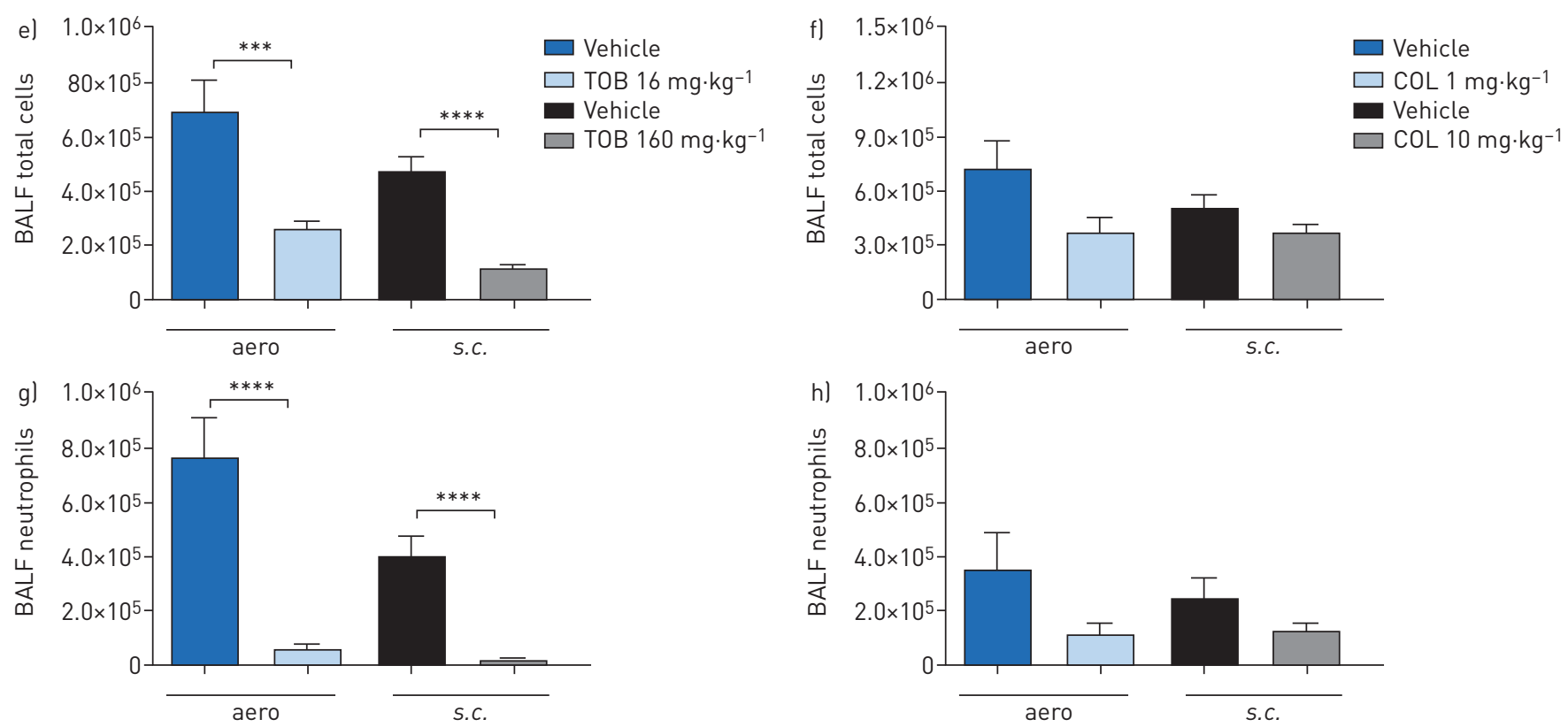

FIGURE 3 Efficacy of "early" treatment with tobramycin (TOB) (a, c, e, g) and colistin (COL) (b, d, f, h) against Pseudomonas aeruginosa MDR-RP73 in a murine model of chronic lung infection. C57BL/6 male mice laged 8-10 weeks) were infected with $5 \times 10^{5}$ colony-forming units (CFUs) of MDR-RP73 embedded in agar beads by intratracheal inoculation. Treatment was started 5 min after infection, with $16 \mathrm{mg} \cdot \mathrm{kg}^{-1} \mathrm{TOB}, 1 \mathrm{mg} \cdot \mathrm{kg}^{-1}$ COL or vehicle administered daily for 7 days via an aerosoliser (aerol. Alternatively, $160 \mathrm{mg} \cdot \mathrm{kg}^{-1} \mathrm{TOB}, 10 \mathrm{mg} \cdot \mathrm{kg}^{-1} \mathrm{COL}$ or vehicle was administered via the subcutaneous (s.c.) route. a, b) Before each administration, mice were weighed, and the percentage change from the initial body weight was averaged for each group of mice. Data are presented as mean士SEM. c-h) At day seven post-infection, mice were killed, bronchoalveolar lavage fluid (BALF) was collected and lungs were excised, homogenised and plated onto tryptic soy agar to determine the bacterial burden (c, d). Each dot represents CFUs per lung from one mouse, and horizontal lines represent the median values. e, f) Total cell and $g, h)$ neutrophil counts were performed in BALF. Data are presented as the mean \pm SEM pooled from two independent experiments $(n=11-16) .^{*}: p<0.05 ;{ }^{* *}: p<0.01 ; * * *: p<0.001$; $* * * *: p<0.0001$.

(aero or i.n.) or systemic (s.c.) routes. Our results showed that aero and s.c. administration of both TOB and COL significantly reduced the bacterial burden. In the acute infection model, efficacy was substantially reduced for both TOB and COL by i.n. administration, highlighting the difficulty of achieving efficacy of even potent drugs with this route. Direct instillations are preferred for local delivery of therapeutic agents into rodent lungs; however, i.n. administration has been reported to result in drug retention in the nasal cavities [21]. These observations are consistent with our PK data and other studies that demonstrated low 

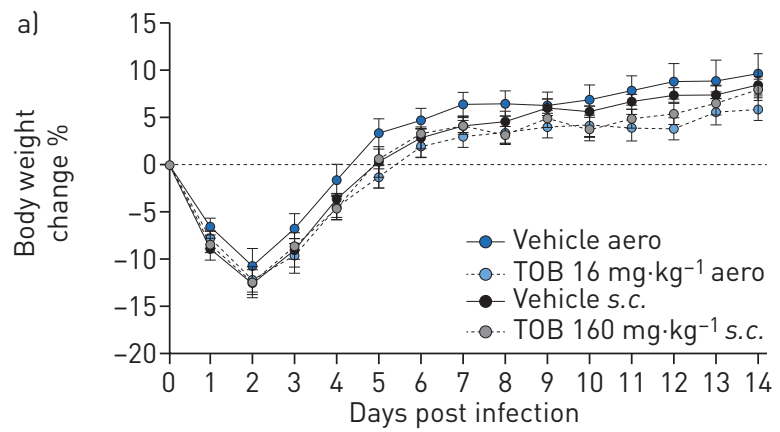

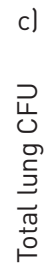

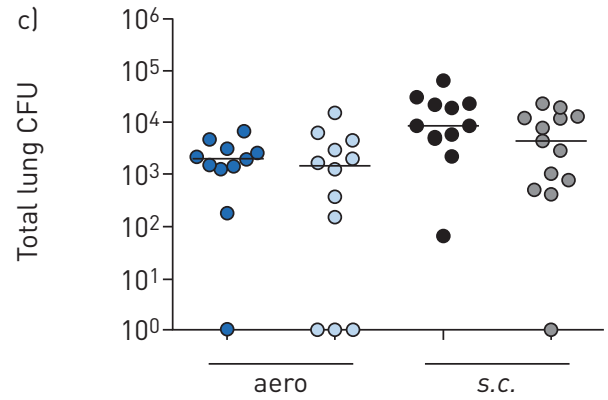

- Vehicle

- TOB $16 \mathrm{mg} \cdot \mathrm{kg}^{-1}$

- Vehicle

- TOB $160 \mathrm{mg} \cdot \mathrm{kg}^{-1}$
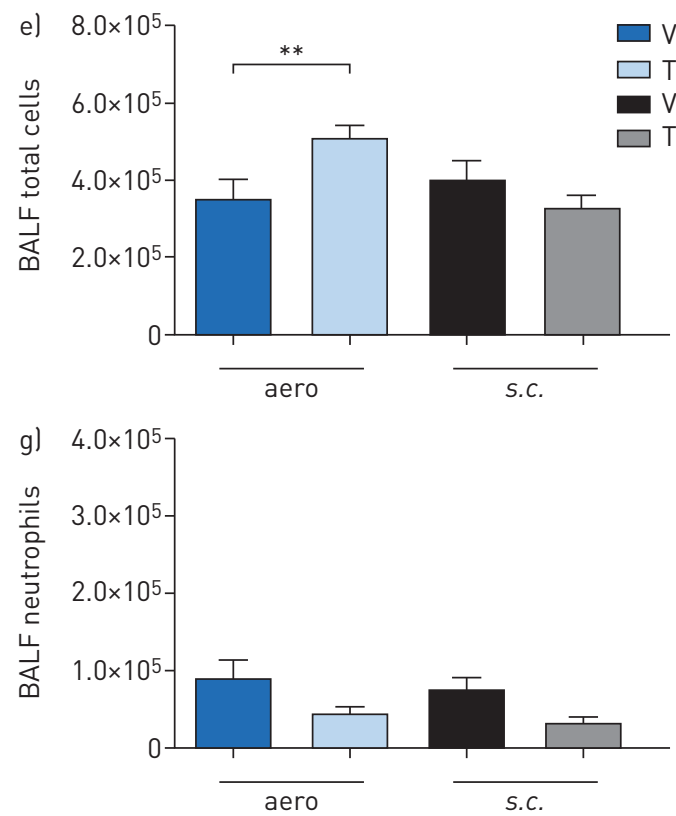
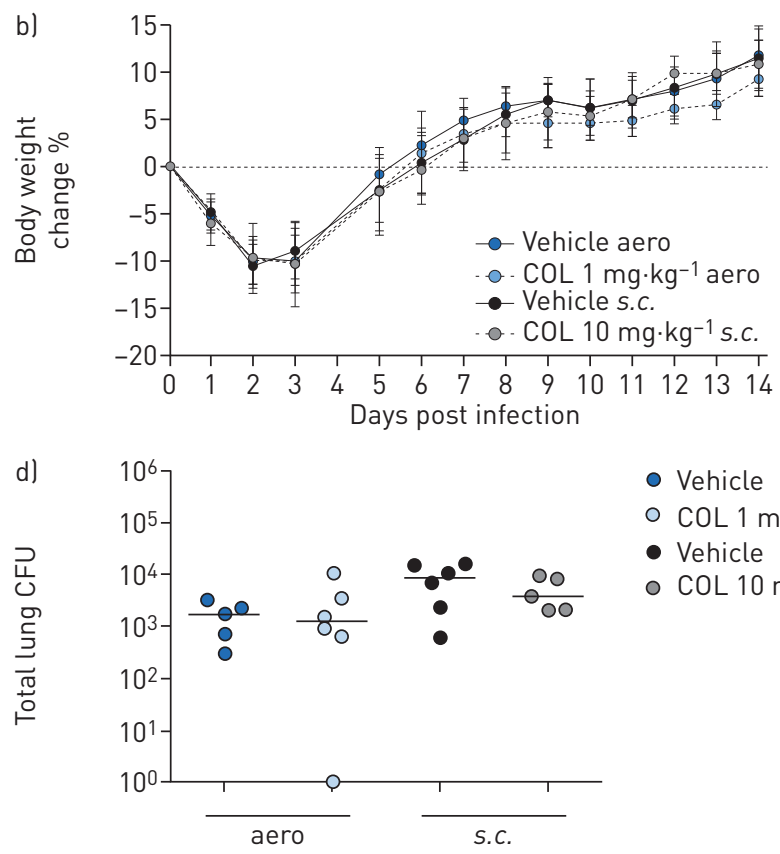

- Vehicle

- COL $1 \mathrm{mg} \cdot \mathrm{kg}^{-1}$

- Vehicle

- COL $10 \mathrm{mg} \cdot \mathrm{kg}^{-1}$
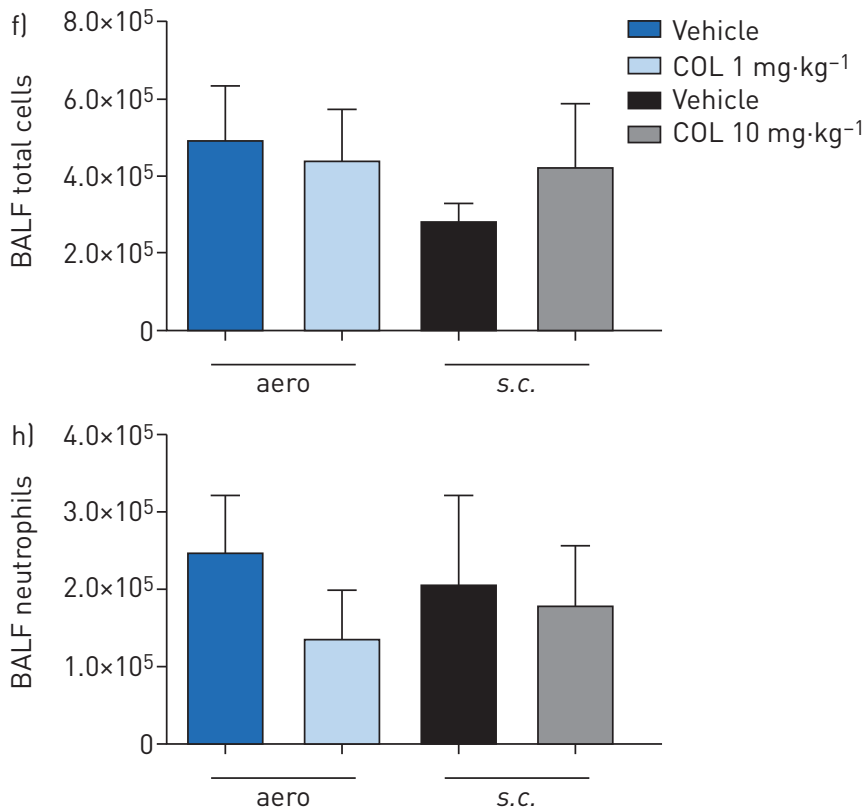

FIGURE 4 Efficacy of "late" treatment with tobramycin (TOB) (a, c, e, g) and colistin (COL) (b, d, f, h) against Pseudomonas aeruginosa MDR-RP73 in a murine model of chronic lung infection. C57BL/6 male mice laged 8-10 weeks) were infected with $5 \times 10^{5}$ colony-forming units (CFUs) of MDR-RP73 embedded in agar beads by intratracheal inoculation. Treatment was started 7 days post-infection and administered daily for another 7 days with $16 \mathrm{mg} \cdot \mathrm{kg}^{-1} \mathrm{TOB}, 1 \mathrm{mg} \cdot \mathrm{kg}^{-1} \mathrm{COL}$ or vehicle via an aerosoliser (aero). Alternatively, $160 \mathrm{mg} \cdot \mathrm{kg}^{-1} \mathrm{TOB}, 10 \mathrm{mg} \cdot \mathrm{kg}^{-1} \mathrm{COL}$ or vehicle was administered via the subcutaneous (s.c.) route. a, b) Before each administration, mice were weighed, and the percentage change from the initial body weight was averaged for each group of mice. Data are presented as mean \pm SEM. $c-h) 7$ days after the beginning of treatment, which is a total of 14 days after infection, the mice were killed, bronchoalveolar lavage fluid (BALF) was collected and lungs were excised, homogenised and plated onto tryptic soy agar to determine the bacterial burden ( $c, d$ ). Each dot represents CFUs per lung from one mouse, and horizontal lines represent the median values. e, f) Total cell and $g, h$ ) neutrophil counts were performed in BALF. Data are presented as the mean \pm SEM pooled from one to two independent experiments $(n=5-14)$. ${ }^{* *}: p<0.01$.

levels of COL in the lung following i.n. administration [22]. In contrast, TOB rapidly reached the airways, indicating its markedly high penetration by i.n. administration. While the use of a conventional human-adapted nebuliser has shown low deposition efficiency and was, therefore, excluded from this work [23], the aerosoliser device used for aero administration is a non-invasive aerosol delivery system that ensures a rapid and good spread of molecules into the airways [24]. This was supported by PK profiles obtained for both TOB and COL following aero administration. Bacterial burden was significantly reduced 
TABLE 2 Cytokine/chemokine concentrations following chronic Pseudomonas aeruginosa airway infection and "early" antibiotic treatment

Concentration $\mathrm{pg} \cdot 500 \mu \mathrm{g}^{-1}$

\begin{tabular}{|c|c|c|c|c|c|c|c|c|}
\hline & Vehicle aero & TOB aero ${ }^{\#}$ & Vehicle s.c. & TOB s.c. " & Vehicle aero & $\mathrm{COL}_{\text {aero }}{ }^{+}$ & Vehicle s.c. & COL s.c. ${ }^{\S}$ \\
\hline IL-1及 & $7.07 \pm 1.07$ & $8.90 \pm 0.74$ & $6.34 \pm 0.41$ & $7.94 \pm 1.07$ & $7.60 \pm 1.12$ & $6.72 \pm 0.78$ & $6.06 \pm 0.24$ & $5.10 \pm 0.42$ \\
\hline IL-3 & $2.24 \pm 0.14$ & $2.52 \pm 0.18$ & $2.44 \pm 0.17$ & $2.89 \pm 0.07$ & $2.36 \pm 0.20$ & $2.45 \pm 0.32$ & $2.13 \pm 0.13$ & $1.86 \pm 0.07$ \\
\hline IL-5 & $2.40 \pm 0.22$ & $2.51 \pm 0.06$ & $2.37 \pm 0.09$ & $3.10 \pm 0.15^{* *}$ & $1.90 \pm 0.28$ & $2.41 \pm 0.42$ & $2.30 \pm 0.16$ & $1.86 \pm 0.10$ \\
\hline IL-6 & $4.88 \pm 0.20$ & $3.72 \pm 0.35 *$ & $4.45 \pm 0.34$ & $3.15 \pm 0.08 *$ & $4.38 \pm 0.43$ & $4.01 \pm 0.56$ & $3.54 \pm 0.16$ & $3.88 \pm 0.10$ \\
\hline IL-12p40 & $68.07 \pm 6.68$ & $37.67 \pm 3.42^{* * *}$ & $59.37 \pm 4.66$ & $32.50 \pm 2.47^{* *}$ & $64.00 \pm 7.71$ & $33.56 \pm 4.20 * *$ & $48.88 \pm 8.11$ & $37.17 \pm 0.71$ \\
\hline IL-12p70 & $26.77 \pm 2.45$ & $20.46 \pm 1.77$ & $26.56 \pm 2.86$ & $22.80 \pm 1.42$ & $19.69 \pm 3.40$ & $19.58 \pm 3.15$ & $20.13 \pm 1.98$ & $17.05 \pm 1.23$ \\
\hline IL-13 & $63.53 \pm 4.62$ & $81.55 \pm 4.38 *$ & $74.97 \pm 3.83$ & $83.52 \pm 3.25$ & $68.18 \pm 5.55$ & $72.57 \pm 5.84$ & $62.75 \pm 2.25$ & $59.91 \pm 3.96$ \\
\hline IL-17A & $15.08 \pm 2.24$ & $6.01 \pm 0.17^{* *}$ & $16.82 \pm 1.37$ & $7.60 \pm 0.82 * *$ & $16.02 \pm 3.68$ & $6.64 \pm 0.80 *$ & $11.98 \pm 0.50$ & $11.04 \pm 1.41$ \\
\hline Eotaxin & $637 \pm 67.26$ & $489 \pm 59.16$ & $634 \pm 31.45$ & $448 \pm 47.34$ & $566 \pm 22.74$ & $439 \pm 17.84^{* *}$ & $534 \pm 41.19$ & $492 \pm 27.48$ \\
\hline G-CSF & $8.50 \pm 0.95$ & $7.26 \pm 0.00$ & $8.26 \pm 0.51$ & $7.26 \pm 0.00$ & $8.87 \pm 1.05$ & $7.26 \pm 0.00$ & $7.34 \pm 0.08$ & $7.26 \pm 0.00$ \\
\hline GM-CSF & $11.82 \pm 1.16$ & $12.22 \pm 1.47$ & $12.28 \pm 0.64$ & $14.61 \pm 0.91$ & $10.59 \pm 1.56$ & $12.10 \pm 1.66$ & $8.92 \pm 1.16$ & $8.80 \pm 0.94$ \\
\hline MIP-1及 & $27.40 \pm 1.99$ & $26.89 \pm 3.08$ & $30.13 \pm 2.78$ & $20.10 \pm 0.89 *$ & $25.59 \pm 1.04$ & $22.49 \pm 1.34$ & $24.24 \pm 1.56$ & $25.19 \pm 1.37$ \\
\hline RANTES & $324 \pm 67.69$ & $69.49 \pm 6.66 * * *$ & $234 \pm 33.58$ & $65.56 \pm 8.55^{*}$ & $173 \pm 25.56$ & $65.29 \pm 6.77^{*}$ & $176 \pm 31.75$ & $147 \pm 31.85$ \\
\hline TNF- $\alpha$ & $44.31 \pm 4.31$ & $34.57 \pm 1.71 *$ & $43.25 \pm 0.31$ & $32.25 \pm 2.79 *$ & $33.59 \pm 2.13$ & $32.79 \pm 4.05$ & $31.41 \pm 1.09$ & $32.12 \pm 2.86$ \\
\hline
\end{tabular}

Data are presented as mean \pm SEM pooled from two independent experiments ( $n=4-5)$. Statistical significance determined by one-way ANOVA with Bonferroni's multiple comparison test, with significant differences between treatment and vehicle highlighted in bold. aero: delivery using an aerosoliser; TOB: tobramycin; s.C.: subcutaneous; COL: colistin; IL: interleukin; G-CSF: granulocyte colony-stimulating factor; GM-CSF: granulocyte-macrophage colony-stimulating factor; IFN: interferon; KC: keratinocyte chemoattractant; MCP: monocyte chemoattractant protein; MIP: macrophage inflammatory protein; TNF: tumour necrosis factor; ${ }^{\#}: 16 \mathrm{mg} \cdot \mathrm{kg}^{-1} ;{ }^{\Uparrow}: 160 \mathrm{mg} \cdot \mathrm{kg}^{-1} ;{ }^{+}: 1 \mathrm{mg} \cdot \mathrm{kg}^{-1} ;{ }^{\S}: 10 \mathrm{mg} \cdot \mathrm{kg}^{-1} ;{ }^{*}:$ $p<0.05 ;^{* *}: p<0.01 ; * * * p<0.001$.

by s.c. administration of both TOB and COL, although lower lung penetration was observed for COL than for TOB. Regarding bacterial eradication, TOB treatment by aero and s.c. administration cleared $P$. aeruginosa in the majority of mice, while COL significantly reduced the bacterial burden, although complete clearance was not achieved. Overall, our data do not fully support any direct correlation between efficacy (CFUs per lung) and drug concentration in the lung (PK), raising the question of whether other drug features or host factors may contribute.

Next, we used a chronic infection model established by direct intratracheal administration of a clinical MDR-RP73 strain embedded in agar beads. Compared to vehicle-treated animals, mice treated by local (aero) or systemic (s.c.) administration of TOB soon after infection ("early" treatment) exhibited significantly increased recovery and gain of body weight and reduced bacterial burden in the lung. In contrast, COL-treated mice did not exhibit differences in recovery and gain of body weight when compared with vehicle-treated mice. In comparison to TOB treatment that resulted in bacterial clearance in most mice when administered via the aero or s.c. routes, only a significant but modest decrease in bacterial burden was observed with COL treatment via the aero route, while s.c. administration of COL was not effective. Thus, "early" treatment with TOB was effective in both the acute and chronic infection models, while the efficacy of COL differed in the two murine models. Toxicity and potential adverse effects of repeated doses may partly explain the results obtained in the chronic infection model for COL $[25,26]$. Our findings suggest that the results seen upon short-term treatment in mouse models of acute infection may not be predictive of the efficacy of the repeated treatment regimen followed in chronic infections.

Our work focused on immunocompetent C57BL/6 mice given that no significant differences have been observed in the bacterial burden between $\mathrm{CF}$ and non-CF mice in previous antibiotic testing procedures [17]. Previous data indicate that the efficacy of treatment is not influenced by the CF environment. By contrast, different bacterial strains can vary the degree of disease severity, potentially impacting on antibiotic efficacy. Our animal experiments were performed with selected $P$. aeruginosa strains, PAO1 for acute infection and MDR-RP73 for chronic infection. This limitation is mitigated somewhat by the 
TABLE 3 Cytokine/chemokine concentrations following long-term chronic Pseudomonas aeruginosa airway infection and "late" antibiotic treatment

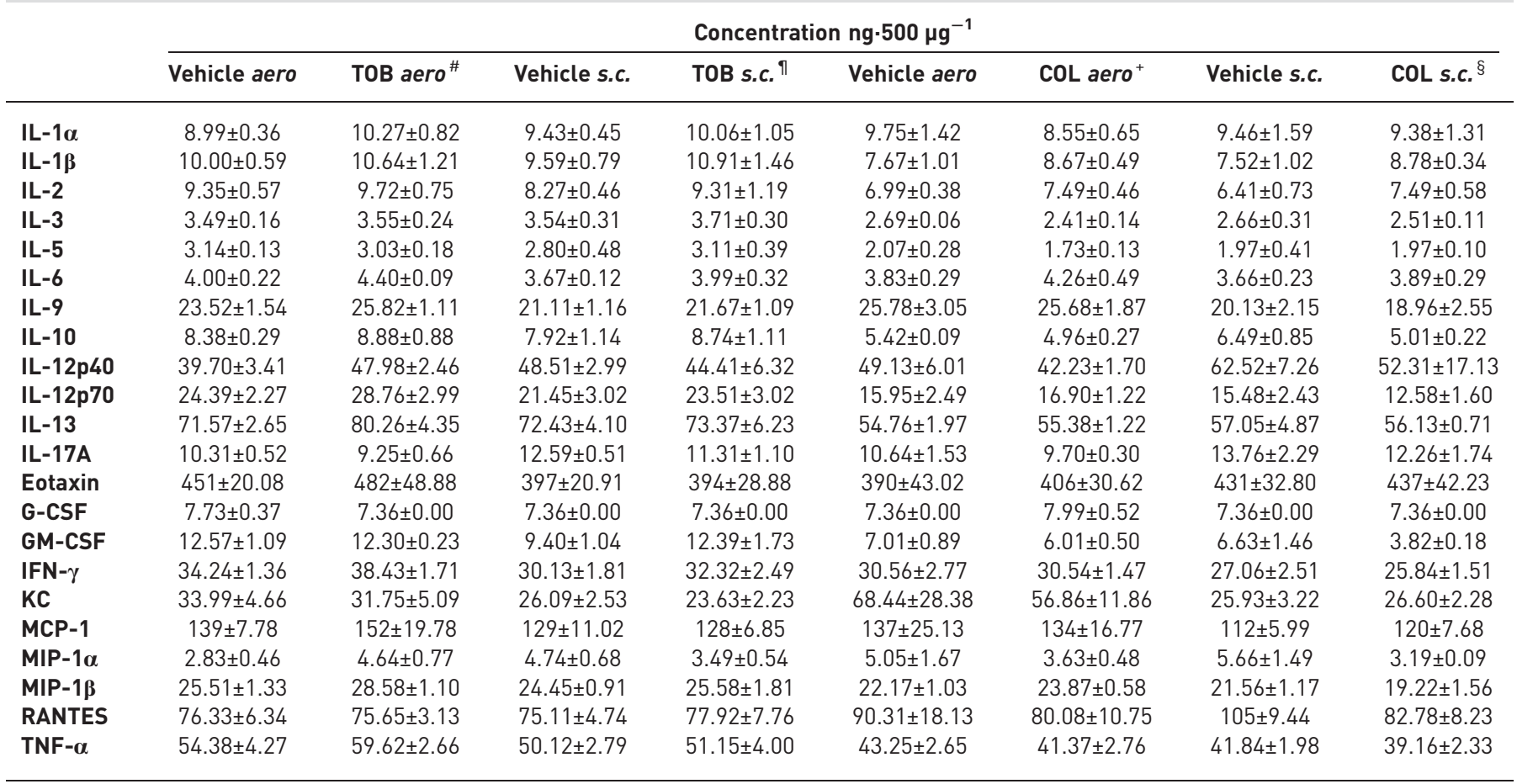

Data are presented as the mean \pm SEM pooled from one to two independent experiments $(n=4-5)$. No statistically significant differences lone-way ANOVA with Bonferroni's multiple comparison test) were found between antibiotics and their respective vehicle. aero: delivery using an aerosoliser; TOB: tobramycin; S.C.: subcutaneous; COL: colistin; IL: interleukin; G-CSF: granulocyte colony-stimulating factor; GM-CSF: granulocyte-macrophage colony-stimulating factor; IFN: interferon; KC: keratinocyte chemoattractant; MCP: monocyte chemoattractant protein; MIP: macrophage inflammatory protein; TNF: tumour necrosis factor; ${ }^{\#}: 16 \mathrm{mg} \cdot \mathrm{kg}^{-1} ;{ }^{\uparrow}: 160 \mathrm{mg} \cdot \mathrm{kg}^{-1} ;{ }^{+}: 1 \mathrm{mg} \cdot \mathrm{kg}^{-1} ;{ }^{\S}: 10 \mathrm{mg} \cdot \mathrm{kg}^{-1}$.

rationale behind this selection. PAO1 is the reference strain endowed with virulence factors most relevant for acute respiratory infections such as hospital-acquired pneumoniae [27, 28]. Among several CF clinical $P$. aeruginosa isolates tested, MDR-RP73 was the most efficient in establishing chronic pneumonia in a murine model $[13,17,29,30]$, reproducing hallmarks of CF and non-CF bronchiectasis.

Antibiotic efficacy is affected by the treatment schedule in chronic infection

In patients with established chronic $P$. aeruginosa pulmonary infection, antibiotics generally fail to eradicate the bacterial pathogen. Thus, rather than eradication, treatment during advanced chronic

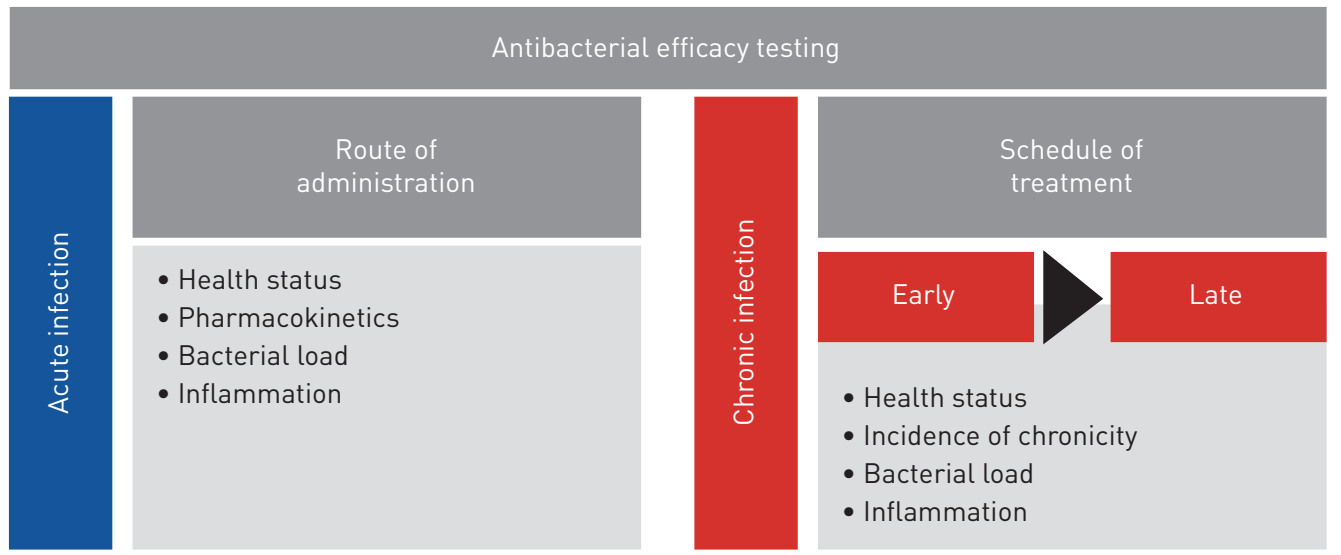

FIGURE 5 Roadmap of antibacterial efficacy testing in mouse models of respiratory infection. The preclinical platform for antibacterial efficacy testing includes acute and chronic infection models and different treatment regimens. Multiple end points, including profiling of bacterial and host responses, are critical parameters to validate the efficacy of anti-Pseudomonas aeruginosa treatment. 
infection focuses on preserving lung function with the resolution of periodic exacerbations [31]. When treatment was started 7 days post-infection ("late" treatment) in the chronic infection model, no reduction in bacterial burden or inflammation, or weight recovery was observed with either TOB or COL. The growth of $P$. aeruginosa in biofilms, which can be observed in our mouse model during advanced chronic lung infection [14], may explain the antibiotic inefficacy, and this phenomenon represents a significant challenge in the treatment of $P$. aeruginosa.

\section{The inflammatory response does not strictly reflect changes in bacterial burden}

This study extended the analysis of infection to multiple host parameters that included measurement of immune cells in the BALF and cytokine/chemokine production in the lung. Although the efficacy of TOB was greater than that of COL in reducing bacterial burden in the acute infection model, COL significantly reduced the inflammatory profile in the airways, particularly the neutrophil load, and to a moderately greater extent than by TOB. This may reflect the contribution of immunomodulatory effects of antibiotics to efficacy [32,33] and deserves further investigation with additional model systems. Assessment of cytokine/chemokine profiles revealed significantly reduced levels of G-CSF and KC in mice treated with TOB and COL by local and systemic administration. Both these immune mediators are known to be determinants of neutrophil production, differentiation and recruitment. Their reduction was independent of the antibiotic and the route of administration, suggesting that G-CSF and KC could be strong candidates for efficacy biomarkers. Other cytokines/chemokines were also affected by the type of antibiotic and the route of administration. It should be pointed out that these differences in host response did not strictly correlate with the extent of bacterial burden modulation.

In chronic pulmonary infection, when treatments were started soon after infection ("early" treatment), TOB was highly effective and attenuated the inflammatory response, particularly of neutrophils, to an even greater extent than in the acute infection model. Concentrations of diverse cytokines/chemokines, such as IL-17A and IL-6, were significantly reduced with both local and systemic administration of TOB, suggesting a complex scenario for the host response. Modulation of other cytokines/chemokines was dependent on the route of administration. For instance, KC was significantly reduced by local treatment, and MIP- $1 \beta$ by systemic treatment. In the case of "early" treatment with COL, the inflammatory response in terms of cells recruited in the BALF was not significantly affected, and only a significant but modest reduction in a few cytokines/chemokines was detected exclusively after local administration.

When treatments were started late after infection ("late" treatment) in the chronic infection model, neither TOB nor COL could decrease the bacterial burden or modulate the immune response. Notably, the entire panel of cytokines/chemokines remained unaffected by the antibiotics, and this result represents a significant concern for the treatment of advanced chronic $P$. aeruginosa infection.

\section{Conclusions}

This work responds to the need for animal models, protocols and end points to improve predictive value and clinical relevance in drug testing in accordance with European Respiratory Society task force recommendations [34]. So far, preclinical animal models do not satisfactorily predict the complexities of human respiratory disease, jeopardising the success of drug efficacy testing. This acquires particular relevance in respiratory infections by $P$. aeruginosa, a pathogen that is notably difficult to treat because of its striking adaptive abilities. Our results in a preclinical platform underline the importance of carefully selecting the appropriate mouse model and treatment regimen for the disease under investigation to optimise antibiotic testing (figure 5). By profiling bacterial and host responses, we defined multiple end points that are critical parameters to validate the efficacy of anti- $P$. aeruginosa treatments. We showed that a combination of these procedures in disease-specific models might provide an enhanced link between preclinical testing and clinical trials. The challenge ahead is to improve these mouse models by including additional variables such as the host genetic background, polymicrobial communities and the concomitant use of other therapies [35-37] to further reflect the complexity of human diseases.

Acknowledgements: The authors would like to thank B. Tümmler (Hannover Medical School, Hannover, Germany) for supplying the $P$. aeruginosa strain from a CF patient. We thank Chiara Bigogno, Ulla Bastrup and Giulio Dondio for PK studies performed by APhaD (Milano, Italy).

Author contributions: Conceiving and designing the experiments: C. Cigana and A. Bragonzi. Performing the experiments: C. Cigana, S. Ranucci, A. Rossi, I. De Fino and M. Melessike. Analysing the data: C. Cigana, S. Ranucci and A. Rossi. Contributing reagents/materials/analysis tools: A. Bragonzi. Writing the paper: C. Cigana and A. Bragonzi.

Conflict of interest: None declared. 
Support statement: Research in A. Bragonzi's laboratory is funded by the Italian Cystic Fibrosis Foundation (CFaCore). The funders had no role in study design, data collection and analysis, decision to publish, or preparation of the manuscript.

\section{References}

1 Ferkol T, Schraufnagel D. The global burden of respiratory disease. Ann Am Thorac Soc 2014; 11: 404-406.

2 Cookson W, Cox MJ, Moffatt MF. New opportunities for managing acute and chronic lung infections. Nat Rev Microbiol 2018; 16: 111-120.

3 Gellatly SL, Hancock RE. Pseudomonas aeruginosa: new insights into pathogenesis and host defenses. Pathog Dis 2013; 67: 159-173.

4 Yonker LM, Cigana C, Hurley BP, et al. Host-pathogen interplay in the respiratory environment of cystic fibrosis. J Cyst Fibros 2015; 14: 431-439.

5 Boucher HW, Talbot GH, Bradley JS, et al. Bad bugs, no drugs: no ESKAPE! An update from the Infectious Diseases Society of America. Clin Infect Dis 2009; 48: 1-12.

6 Mogayzel PJ Jr, Naureckas ET, Robinson KA, et al. Cystic Fibrosis Foundation pulmonary guideline. Pharmacologic approaches to prevention and eradication of initial Pseudomonas aeruginosa infection. Ann Am Thorac Soc 2014; 11: 1640-1650.

7 Moradali MF, Ghods S, Rehm BH. Pseudomonas aeruginosa lifestyle: a paradigm for adaptation, survival, and persistence. Front Cell Infect Microbiol 2017; 7: 39.

8 Zak O, O'Reilly T. Animal models as predictors of the safety and efficacy of antibiotics. Eur J Clin Microbiol Infect Dis 1990; 9: 472-478.

9 Zak O, O'Reilly T. Animal models in the evaluation of antimicrobial agents. Antimicrob Agents Chemother 1991; 35: 1527-1531.

10 Bragonzi A. Murine models of acute and chronic lung infection with cystic fibrosis pathogens. Int J Med Microbiol 2010; 300: 584-593.

11 Bragonzi A. Fighting back: peptidomimetics as a new weapon in the battle against antibiotic resistance. Sci Transl Med 2010; 2: 21ps29.

12 Bragonzi A, Paroni M, Nonis A, et al. Pseudomonas aeruginosa microevolution during cystic fibrosis lung infection establishes clones with adapted virulence. Am J Respir Crit Care Med 2009; 180: 138-145.

13 Facchini M, De Fino I, Riva C, et al. Long term chronic Pseudomonas aeruginosa airway infection in mice. J Vis Exp 2014; 85: e51019.

14 Cigana C, Lore NI, Riva C, et al. Tracking the immunopathological response to Pseudomonas aeruginosa during respiratory infections. Sci Rep 2016; 6: 21465.

15 Lore NI, Cigana C, Riva C, et al. IL-17A impairs host tolerance during airway chronic infection by Pseudomonas aeruginosa. Sci Rep 2016; 6: 25937.

16 Bragonzi A, Worlitzsch D, Pier GB, et al. Nonmucoid Pseudomonas aeruginosa expresses alginate in the lungs of patients with cystic fibrosis and in a mouse model. J Infect Dis 2005; 192: 410-419.

17 Cigana C, Bernardini F, Facchini M, et al. Efficacy of the novel antibiotic POL7001 in preclinical models of Pseudomonas aeruginosa pneumonia. Antimicrob Agents Chemother 2016; 60: 4991-5000.

18 Kukavica-Ibrulj I, Facchini M, Cigana C, et al. Assessing Pseudomonas aeruginosa virulence and the host response using murine models of acute and chronic lung infection. Methods Mol Biol 2014; 1149: 757-771.

19 Moalli F, Paroni M, Véliz Rodriguez T, et al. The therapeutic potential of the humoral pattern recognition molecule PTX3 in chronic lung infection caused by Pseudomonas aeruginosa. J Immunol 2011; 186: 5425-5434.

20 Kilkenny C, Browne WJ, Cuthill IC, et al. Improving bioscience research reporting: the ARRIVE guidelines for reporting animal research. PLoS Biol 2010; 8: e1000412.

21 Fernandes CA, Vanbever R. Preclinical models for pulmonary drug delivery. Expert Opin Drug Del 2009; 6: 1231-1245.

22 Lin YW, Zhou QT, Cheah SE, et al. Pharmacokinetics/pharmacodynamics of pulmonary delivery of colistin against Pseudomonas aeruginosa in a mouse lung infection model. Antimicrob Agents Chemother 2017; 61: e02025-16.

23 Stangl R. A nebulizer for rodent inhalation studies. Respir Drug Deliv 2008: 697-700.

24 Bivas-Benita M, Zwier R, Junginger $\mathrm{HE}$, et al. Non-invasive pulmonary aerosol delivery in mice by the endotracheal route. Eur J Pharm Biopharm 2005; 61: 214-218.

25 Pogue JM, Lee J, Marchaim D, et al. Incidence of and risk factors for colistin-associated nephrotoxicity in a large academic health system. Clin Infect Dis 2011; 53: 879-884.

26 Ahmed MU, Velkov T, Lin YW, et al. Potential toxicity of polymyxins in human lung epithelial cells. Antimicrob Agents Chemother 2017; 61: e02690-16.

27 Stover CK, Pham XQ, Erwin AL, et al. Complete genome sequence of Pseudomonas aeruginosa PA01, an opportunistic pathogen. Nature 2000; 406: 959-964.

28 Dubern J, Cigana C, De Simone M, et al. Integrated whole-genome screening for Pseudomonas aeruginosa virulence genes using multiple disease models reveals that pathogenicity is host specific. Environ Microbiol 2015; 17: 4379-4393.

29 Jeukens J, Boyle B, Bianconi I, et al. Complete genome sequence of persistent cystic fibrosis isolate Pseudomonas aeruginosa strain RP73. Genome Announc 2013; 1: e00568-13.

30 Bianconi I, Jeukens J, Freschi L, et al. Comparative genomics and biological characterization of sequential Pseudomonas aeruginosa isolates from persistent airways infection. BMC Genomics 2015; 16: 1105.

31 Rogers GB, Hoffman LR, Doring G. Novel concepts in evaluating antimicrobial therapy for bacterial lung infections in patients with cystic fibrosis. J Cyst Fibros 2011; 10: 387-400.

32 Gziut M, MacGregor HJ, Nevell TG, et al. Anti-inflammatory effects of tobramycin and a copper-tobramycin complex with superoxide dismutase-like activity. Br J Pharmacol 2013; 168: 1165-1181.

33 Matzneller P, Strommer S, Drucker C, et al. Colistin reduces LPS-triggered inflammation in a human sepsis model in vivo: a randomized controlled trial. Clin Pharmacol Ther 2017; 101: 773-781.

34 Bonniaud P, Fabre A, Frossard N, et al. Optimising experimental research in respiratory diseases: an ERS statement. Eur Respir J 2018; 51: 1702133. 
35 Relling MV, Evans WE. Pharmacogenomics in the clinic. Nature 2015; 526: 343-350.

36 Rogers GB, Hoffman LR, Whiteley M, et al. Revealing the dynamics of polymicrobial infections: implications for antibiotic therapy. Trends Microbiol 2010; 18: 357-364.

37 Brochado AR, Telzerow A, Bobonis J, et al. Species-specific activity of antibacterial drug combinations. Nature 2018; 559: 259-263. 